\title{
Role of MFS Transporter QDR2 in a Pathogen Candida glabrata
}

Tria Widiasih Widiyanto $^{1}$, Xinyue Chen ${ }^{1}$, Shun Iwatani ${ }^{1}$, Hiroji Chibana ${ }^{2}$, Susumu Kajiwara ${ }^{1}$ ${ }^{1}$ School of Life Science and Technology, Tokyo Institute of Technology, J3-1017 4259 Nagatsuta-cho, Midori-ku, Yokohama, Kanagawa, 226-8503 Japan

${ }^{2}$ Medical Mycology Research Center, Chiba University, 1-8-1 Inohana, Chuo-ku, Chiba, 260-8673 Japan

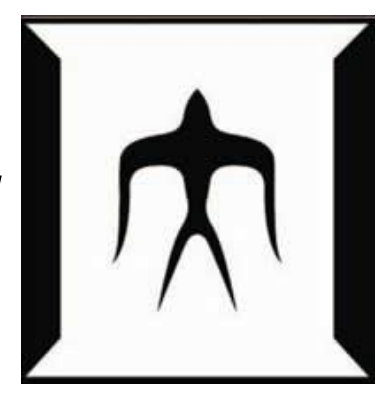

Abstract Candida glabrata is one of the commensal fungal species in human. In immune compromised individuals, it becomes one of the second major causes of candidiasis in mucosal, blood stream, and genito-urinary tract. The high incidences of $C$. glabrata infections have increased significantly in the last two decades, mainly due to its abilities to resist various antifungal drugs (MDR) and form biofilm. The ability of membrane transport to actively efflux the drug out of the cell is mediated mainly by ATP-Binding Cassette (ABC) and MFS (Major Facilitator Superfamily) transporters. In this study, we focus on one of $C$. glabrata genes putatively belong to MFS-DHA1 family, namely $C$. glabrata QDR2. This gene was identified as one of the genes related to the biofilm formation in our previous study. By forming the biofilm on implanted medical devices, C. glabrata limits drug penetration and further cause drug resistance with persistent infection. In an attempt to clarify the mechanism of $C$. glabrata biofilm formation, we investigated the role of CgQDR2 in physiological traits of $C$. glabrata. Based on our results, we suggested that QDR2 deletion might cause an impaired ability of $C$. glabrata to maintain $\mathrm{pH}$ homeostasis, then might lead to the reduction of cell growth, adherent cells numbers, the amount of biofilm formed, and eventually affect $C$. glabrata biofilm susceptibility and drug efflux towards antifungal drugs.

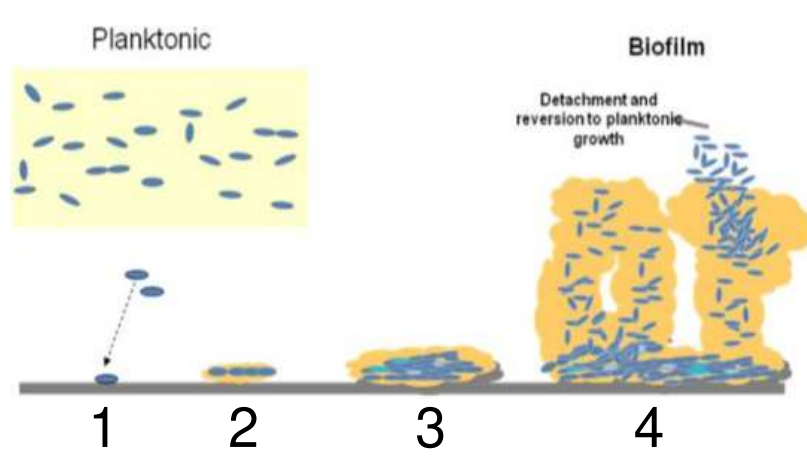

Figure 1. Illustration of biofilm formation starting with (1) adhesion, (2-3) Proliferation \& Colonization, (4) Biofilm Maturation and Cell Detachment/Dispersal (Rangathan, 2014)

\section{QDR2 Biofilm Formation and Expression Level}

CgQDR2, a DHA1-MFS membrane transporter, might be involved in $C$. glabrata metabolism and biofilm formation.

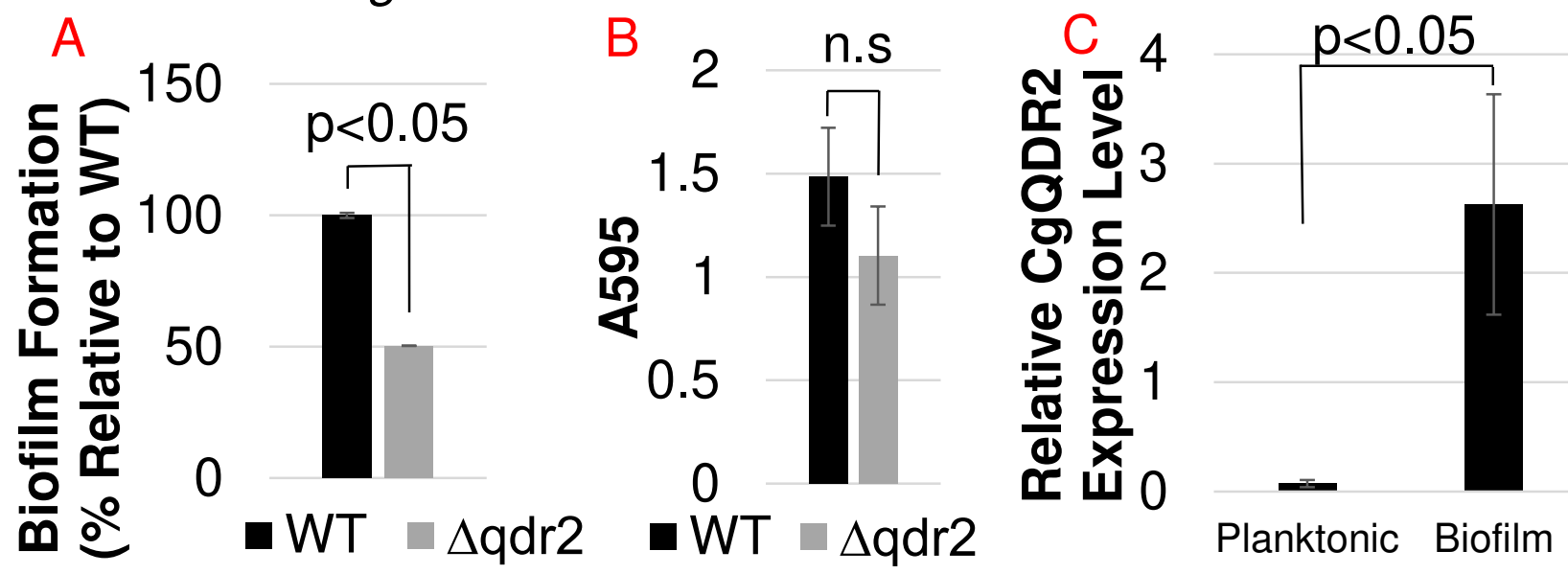

Figure 2. Biofilm formation ( $24 \mathrm{~h}$ ) of $C$. glabrata $\Delta q d r 2$ with relative to $C$. glabrata CBS138 wild type strain assessed with XTT (living cells) (A), Crystal Violet (bulk bofilm) (B), and relative QDR2 mRNA expression level of C. glabrata CBS138 wild type strain during planktonic and biofilm phase (C)

Scanning Electron Microscope (SEM) Analysis of $C$. glabrata WT and $\Delta q d r 2$ Biofilm

Less adhesion area, less extracellular matrix (ECM), and irregular cell shapes were observed from C. glabrata $\Delta q d r 2$ biofilm as compared to WT.

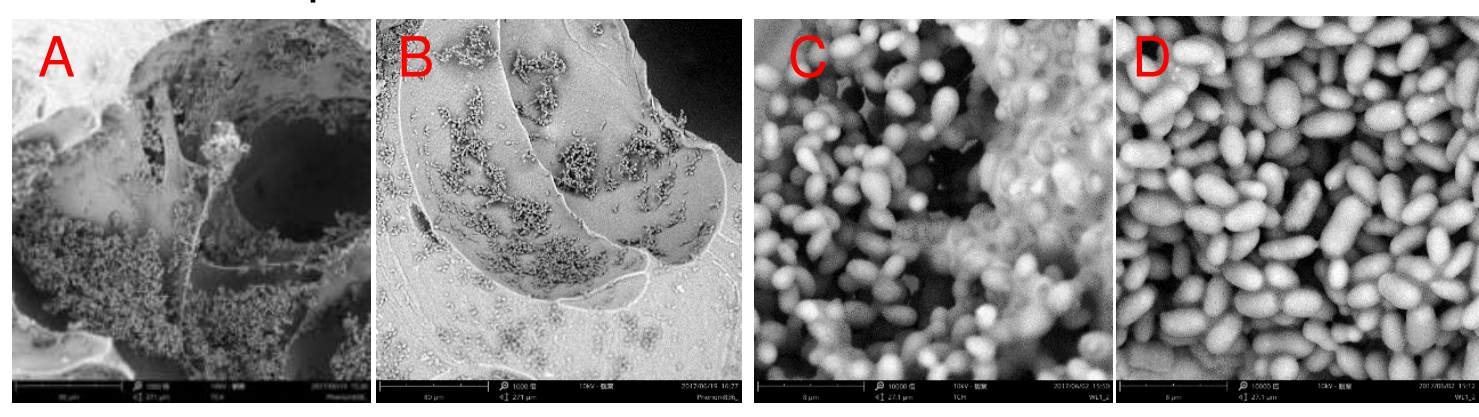

Figure 3. SEM images of $C$. glabrata biofilm with 1000x magnification of CBS138 wild type strain (A) and $\Delta q d r 2(B)$ and $10000 x$ magnification of CBS138 wild type strain (C) and $\Delta q d r 2(D)$ on $9 \mathrm{~mm}^{3}$ cubical silicone sponge.

\section{Fluconazole Susceptibility Assay During Biofilm Formation}

QDR2 deletion affected C. glabrata susceptibility to fluconazole, that might interrupt the drug efflux mechanism in C. glabrata biofilm.

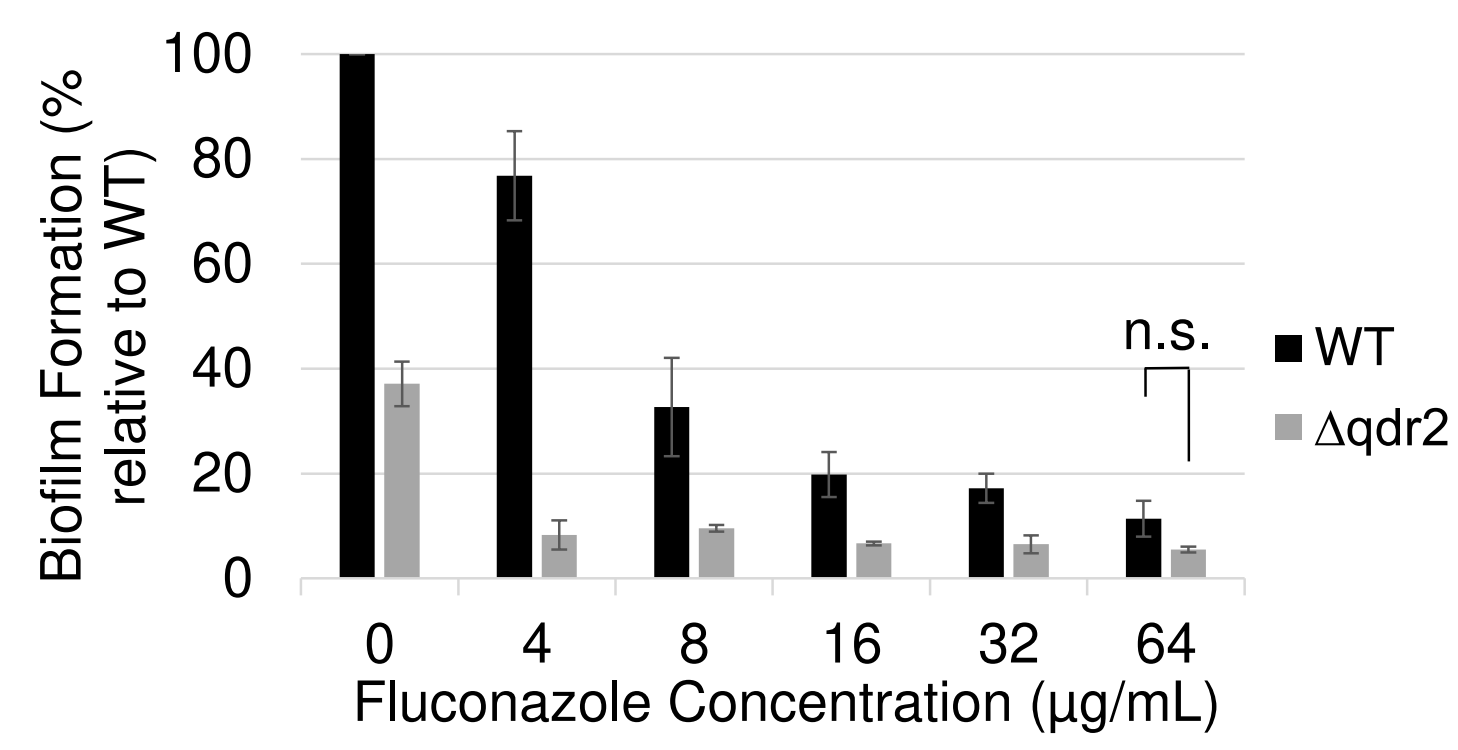

Figure 4. Fluconazole drug susceptibility of C. glabrata biofilm $(24 \mathrm{~h})$ assessed with XTT assay with \% relative to C. glabrata CBS138 wild type, assumed as metabolically active cells

\section{Adhesion Ability}

QDR2 deletion could affect the critical first stage of the biofilm formation process in C. glabrata.

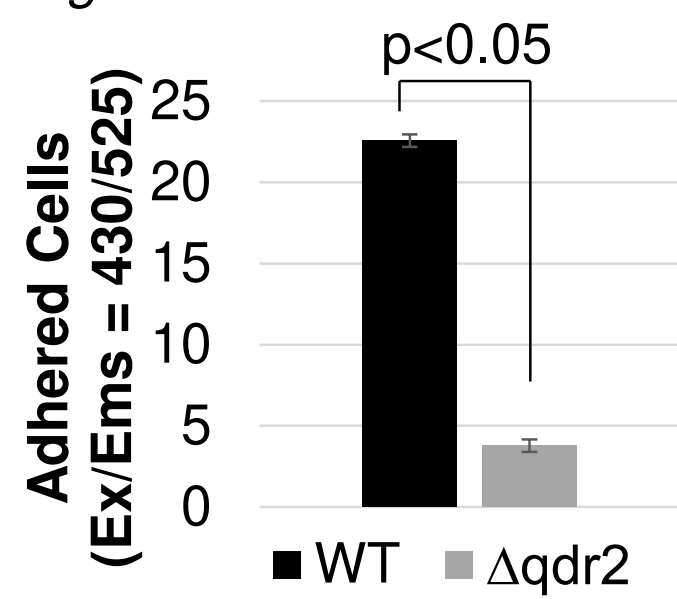

Figure 5. Adhered cells $(90 \mathrm{~min})$ of C. glabrata $\triangle q d r 2$ with relative to $C$ glabrata CBS138 wild type strain assessed with CFDA-SE

\section{C. glabrata $\Delta \mathrm{qdr2} \mathrm{pH}$ Regulation}

QDR2 deletion may affect RIM101 expression with a possibility of $C$. glabrata $\mathrm{pH}$ homeostasis strategy.

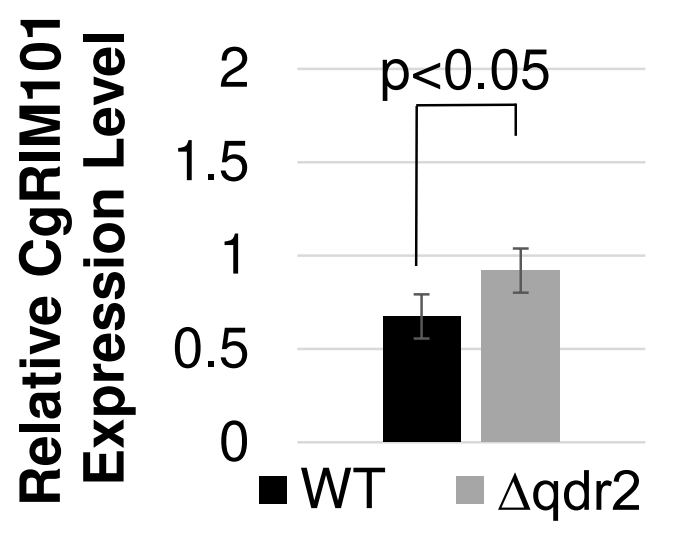

Figure 6. Relative RIM101 mRNA CBS138 wild type strain and $C$ glabrata $\Delta q d r 2$ planktonic phase expression level of $C$. glabrata

\section{C. glabrata $\triangle \mathrm{qdr} 2 \mathrm{pH}$ Regulation}

The loss of QDR2 has altered its intracellular $\mathrm{pH}$ and caused $C$. glabrata unable to grow in neutral conditions, implicating its role in $\mathrm{pH}$ regulation.

Table 1. Intracellular $\mathrm{pH}$ of C.glabrata wild type, mutant, and S. cerevisiae, as comparison, in CSM-0.1 M HEPES pH 6

\begin{tabular}{|l|c|}
\hline CSM-0.1 M HEPES pH 6 & pHi \\
\hline S. cerevisiae S288c & $6.77 \pm 0.19$ \\
\hline C. glabrata WT & $6.63 \pm 0.31$ \\
\hline C. glabrata qdr2 mutant & $4.53 \pm 0.26$ \\
\hline
\end{tabular}

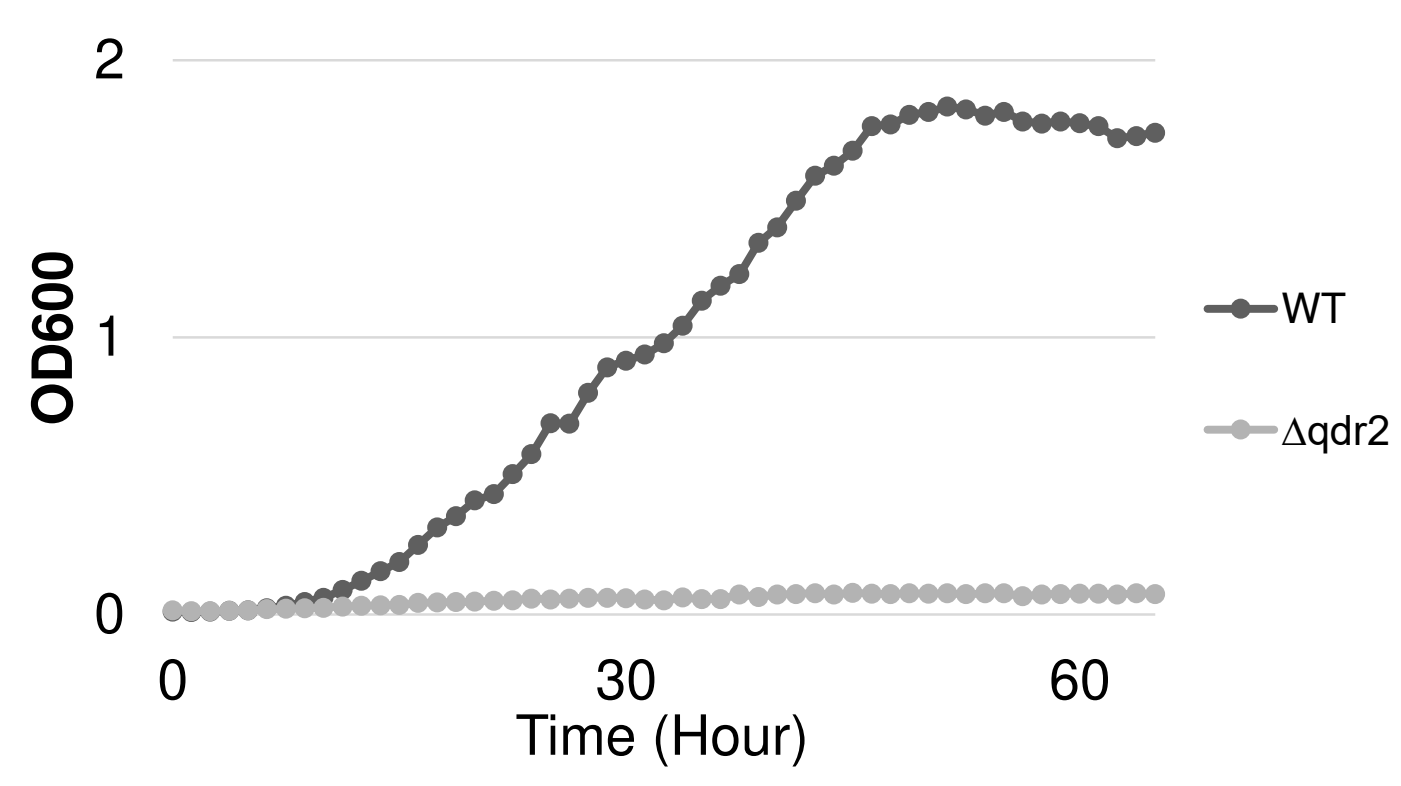

Figure 7. Growth of C. glabrata CBS138 wild type strain and $\Delta q d r 2$ in CSM-His buffered with $0.05 \mathrm{M}$ citric acid- $\mathrm{Na}_{2} \mathrm{HPO}_{4} \mathrm{pH} 6.8$ 